\title{
IDENTIFICATION OF FAULTS IN NONLINEAR DYNAMIC SYSTEMS
}

\author{
Vladimir Filaretov, Alexey Zhirabok, Alexander Zuev, Alexander Protcenko \\ Far Eastern Federal University, 8 Suhanova St., Vladivostok 690950, Russia \\ Institute for Automation and Control Processes FEB RAS, 5 Radio St., Vladivostok 690041, Russia
}

\begin{abstract}
This paper considers a problem of fault detection, isolation and identification of its values in mechatronic systems described by nonlinear dynamic models. To solve this problem logic-dynamic approach and special feedback by residual signal is suggested. This approach consists of three main steps: replacing the initial nonlinear system by certain linear logic-dynamic system, obtaining the bank of linear logic-dynamic observers, and transforming these observes into the nonlinear ones. Logic-dynamic approach allows detecting and isolating faults in nonlinear dynamic systems by using the linear methods for diagnosis in nonlinear mechatronic systems. Feedback of special form introduced in the observers after the detection and isolation of faults. It is allows to determine the value of faults even in nonlinear systems with incomplete observability. The results of mathematical simulation fully confirm the efficiency and high performance of the proposed method.
\end{abstract}

Keywords: mechatronic systems; nonlinear models; fault detection; isolation; identification; observers
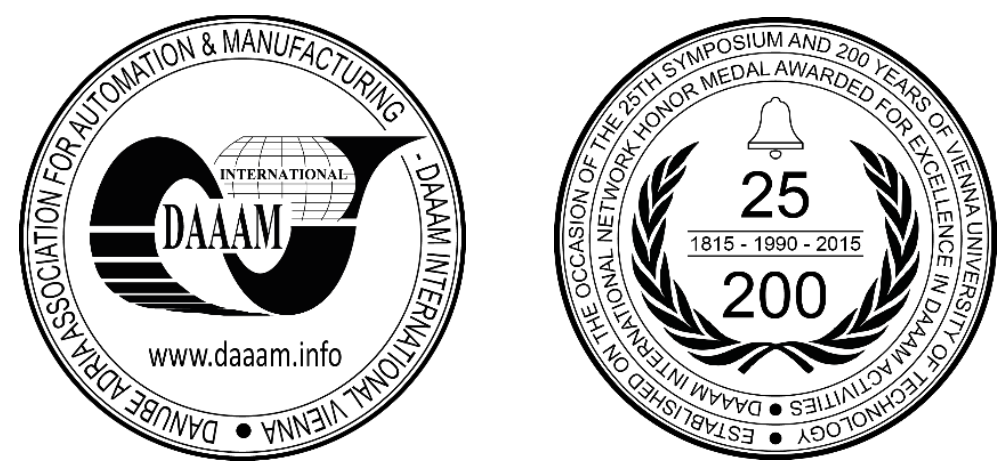

This Publication has to be referred as: Filaretov, V[ladimir]; Zhirabok, A[lexey]; Zuev, A[lexander] \& Protcenko, A[leksandr] (2016). Identification of Faults in Nonlinear Dynamic Systems, Proceedings of the 26th DAAAM International Symposium, pp.0470-0477, B. Katalinic (Ed.), Published by DAAAM International, ISBN 978-3-90273407-5, ISSN 1726-9679, Vienna, Austria

DOI: $10.2507 / 26$ th.daaam.proceedings.062 


\section{Introduction}

One of the possibilities to improve the efficiency of operation of technical systems for critical applications it is operative detection and localization of faults that may occur in elements and subsystems in the process of operation. Timely detection, localization and identification of the values of these faults allow the use of fault-tolerant control methods for the formation of specific control signals that allow to keep the most important characteristics of the system in the presence of faults in it (possibly with a deterioration of the secondary characteristics).

Methods of technical diagnostics are commonly used for the detection and localization of faults. Some of the most widely used methods use the concept of analytical redundancy. This concept presupposes the existence of two or more ways of determining variables of the system. In this case, the diagnosis includes the steps of forming the residual and decision-making. The residual is the result of a mismatch between the behavior of a real diagnosed system and the behavior of its mathematical model (diagnostic observer).

Several methods for synthesis of diagnostic observers for nonlinear dynamical systems are known, including those based on the differential geometric approach [4], the algebra of functions [5, 6], logic-dynamic approach [7, 8, 10] and others $[9,11,12,13]$. The first two allow obtaining the optimal solution with a minimum dimension of observer, however, have a rather complicated process of finding a solution, because of complex analytical calculations. Logicdynamic approach does not guarantee a minimum dimension, but the process of finding a solution on its basis is quite simple because of using of linear methods. The task of identifying the values of the faults on the basis of logic-dynamic approach has been solved in [2] and [3], by introducing into the diagnostic observers the signal of feedback by the residual. However, this approach is valid only for the case when the state vector of the diagnosing object fully observable and diagnostic observers has the first order.

In this paper a special feedback by the signal of residual is introduced in the diagnostic observers obtained with the logic-dynamic approach to solve the problem of identification of values of faults. It is allow getting an accurate estimate of the faults occurring in multidimensional nonlinear systems, including in the case of partial observability of the state vector of the object.

\section{Model of diagnosed object}

In general, non-linear multidimensional diagnosed object (DO) can be described by a model of the form:

$$
\begin{aligned}
\dot{\boldsymbol{x}}(t) & =\boldsymbol{F} \boldsymbol{x}(t)+\boldsymbol{B}(\boldsymbol{x}(t), \boldsymbol{u}(t))+\boldsymbol{G u}(t)+\boldsymbol{L} \boldsymbol{d}(t) \\
\boldsymbol{y}(t) & =\boldsymbol{H} \boldsymbol{x}(t)
\end{aligned}
$$

where $\boldsymbol{x} \in R^{n}$ is state vector, $n$ is dimensionality of DO, $\boldsymbol{y} \in R^{m}$ is output vector, $\boldsymbol{u} \in R^{l}$ is input vector, p-dimensional vector $\boldsymbol{d}(t)$ describes the errors occurring in the system because of the appearance of faults in it (in the absence of faults all the elements of the vector are zero; in the presence of a fault corresponding element of the vector becomes unknown function of time). $\boldsymbol{F} \in R^{n \times n}, \boldsymbol{G} \in R^{n \times l}, \boldsymbol{H} \in R^{m \times n}$ are known matrixes. $\boldsymbol{B}(\boldsymbol{x}(t), \boldsymbol{u}(t))$ is a vector which determines a non-linear part of the system. In this paper a case where each of the di elements of vector $d(t)$ included only in one of the equations of (1) is considered, which means that there is only one nonzero element in each of the columns of matrix $\boldsymbol{L} \in R^{n \times p}$.

The task of detecting faults and determining their values are invited to decide on the basis of logic-dynamic approach (LDA) [1], which allows the construction of diagnostic observers using only linear methods. After synthesis of the diagnostic observers, the special feedback must be introduced in them to ensure stability and to determine the value of the faults occurring in the system.

The developed method provides a set of nonlinear diagnostic observers, each of which is sensitive to one of the faults and invariant to the others. The output signals of observers are the values of the corresponding faults.

As a result of the well-known procedure of synthesis of diagnostic observers based on the LDA [1] k-dimensional observer sensitive to one of the faults can be built in the following form:

$$
\begin{aligned}
\dot{\boldsymbol{x}}^{*}(t) & =\boldsymbol{F}^{*} \boldsymbol{x}^{*}(t)+\boldsymbol{B}^{*}\left(\boldsymbol{x}^{*}(t), \boldsymbol{u}(t)\right)+\boldsymbol{G}^{*} \boldsymbol{u}(t)+\boldsymbol{J} \boldsymbol{y}(t) \\
\boldsymbol{y}^{*}(t) & =\boldsymbol{H}^{*} \boldsymbol{x}^{*}(t)
\end{aligned}
$$

where $\mathrm{x}^{*}$ is state vector of observer, $\mathrm{y}^{*}$ is output signal of observer, $\boldsymbol{F}^{*} \in R^{k \times k}, \boldsymbol{G}^{*} \in R^{k \times l}, \boldsymbol{H}^{*} \in R^{1 \times n}$ are matrixes to be determined [1]. On the basis of the outputs of DO and observer following residual is generated:

$$
r(t)=\boldsymbol{C y}(t)-\boldsymbol{y}^{*}(t)
$$


where $\mathrm{C}$ is $m$-dimensional vector.

The state vectors of DO and observer are associated by the matrix $\boldsymbol{\Phi} \in R^{k \times n}$ [1], and, in the absence of a mismatch between the DO and the observer (if $r=0$ ), satisfy the following equation:

$$
\boldsymbol{x}^{*}=\boldsymbol{\Phi} \boldsymbol{x}
$$

In case of any fault the residual (3) becomes different from zero, and the equation (4) is no longer fulfilled.

To solve the problem of identification of faults, consider how residual will change in the event of faults. After differentiating the expression (3) taking into account (1) and (2) it is can be obtained:

$$
\begin{aligned}
& \dot{r}=\mathbf{C H}(\mathbf{F x}+\mathbf{G u}+\mathbf{B}(\mathbf{x}, \mathbf{u})+\mathbf{L d})-\mathbf{H}^{*}\left(\mathbf{F}^{*} \mathbf{x}^{*}+\mathbf{G}^{*} \mathbf{u}+\mathbf{B}^{*}\left(\mathbf{x}^{*}, \mathbf{u}\right)+\mathbf{J y}\right), \\
& \dot{r}=\boldsymbol{C H F x}+\boldsymbol{C H G u}+\boldsymbol{C H B}(\boldsymbol{x}, \boldsymbol{u})+\boldsymbol{C H L d}-\boldsymbol{H}^{*} \boldsymbol{F}^{*} \boldsymbol{x}^{*}-\boldsymbol{H}^{*} \boldsymbol{G}^{*} \boldsymbol{u}-\boldsymbol{H}^{*} \boldsymbol{B}^{*}\left(\boldsymbol{x}^{*}, \boldsymbol{u}\right)-\boldsymbol{H}^{*} \boldsymbol{J} \boldsymbol{y} .
\end{aligned}
$$

In view of this, as well as the known relations [1] $\boldsymbol{G}^{*}=\boldsymbol{\Phi} \boldsymbol{G}, \boldsymbol{C H}=\boldsymbol{H}^{*} \boldsymbol{\Phi}, \boldsymbol{\Phi} \boldsymbol{F}=\boldsymbol{F}^{*} \boldsymbol{\Phi}+\boldsymbol{J} \boldsymbol{H}, \quad$ it can be obtained:

$$
\begin{aligned}
& \dot{r}=H^{*} \Phi \boldsymbol{F} \boldsymbol{x}-\boldsymbol{H}^{*} \boldsymbol{F}^{*} \boldsymbol{x}^{*}-\boldsymbol{H}^{*} \boldsymbol{J} \boldsymbol{H} \boldsymbol{x}+\boldsymbol{H}^{*} \Phi B(\boldsymbol{x}, \boldsymbol{u})-\boldsymbol{H}^{*} \boldsymbol{B}^{*}\left(\boldsymbol{x}^{*}, \boldsymbol{u}\right)+\boldsymbol{H}^{*} \Phi G \boldsymbol{G}-\boldsymbol{H}^{*} \boldsymbol{G}^{*} \boldsymbol{u}+\boldsymbol{H}^{*} \Phi L d \\
& \dot{r}=\boldsymbol{H}^{*}\left(\boldsymbol{F}^{*}\left(\Phi \boldsymbol{x}-\boldsymbol{x}^{*}\right)+\Phi B(\boldsymbol{x}, \boldsymbol{u})-\boldsymbol{B}^{*}\left(\boldsymbol{x}^{*}, \boldsymbol{u}\right)+\Phi L d\right)
\end{aligned}
$$

Denote the value of $\boldsymbol{\Phi} \boldsymbol{x}-\boldsymbol{x}^{*}$ as a vector of mismatch of states of DO and the observer:

$$
\boldsymbol{e}=\boldsymbol{\Phi} \boldsymbol{x}-\boldsymbol{x}^{*}
$$

Considering introduced vector, it can be obtained:

$$
\dot{r}=\boldsymbol{H}^{*}\left(\boldsymbol{F}^{*} \boldsymbol{e}+\boldsymbol{\Phi B}(\boldsymbol{x}, \boldsymbol{u})-\boldsymbol{B}^{*}\left(\boldsymbol{x}^{*}, \boldsymbol{u}\right)+\boldsymbol{\Phi} \boldsymbol{L} d\right)
$$

Consider the relationship of the vector of mismatch e with the residual $r$. In view of (3) it can be obtained:

$$
r=\boldsymbol{C y}-\boldsymbol{y}^{*}=\boldsymbol{C H} \boldsymbol{x}-\boldsymbol{H}^{*} \boldsymbol{x}^{*}=\boldsymbol{H}^{*} \boldsymbol{\Phi} \boldsymbol{x}-\boldsymbol{H}^{*} \boldsymbol{x}^{*}=\boldsymbol{H}^{*} \boldsymbol{e}
$$

From [1] is known that:

$$
\boldsymbol{F}^{*}=\left[\begin{array}{ccccc}
0 & 1 & 0 & \ldots & 0 \\
0 & 0 & 1 & \ldots & 0 \\
\ldots & \ldots & \ldots & \ldots & \ldots \\
0 & 0 & 0 & \ldots & 0
\end{array}\right], \quad \boldsymbol{H}^{*}=\left[\begin{array}{lllll}
1 & 0 & 0 & \ldots & 0
\end{array}\right]
$$

Thus, taking into account the form of vector $\mathrm{H}^{*}$, the residual $\mathrm{r}$ is the first element of mismatch vector e:

$$
r=e_{1}
$$

Then, taking into account (6) and (7) it can be obtained:

$$
\dot{\boldsymbol{e}}=\boldsymbol{F}^{*} \boldsymbol{e}+\boldsymbol{\Phi} \boldsymbol{B}(\boldsymbol{x}(t), \boldsymbol{u}(t))-\boldsymbol{B}^{*}\left(\boldsymbol{x}^{*}(t), \boldsymbol{u}(t)\right)+\boldsymbol{\Phi} \boldsymbol{L} \boldsymbol{d}
$$

From (9) it follows that the change of the mismatch vector depends on the element $\boldsymbol{\Phi} \boldsymbol{B}(\boldsymbol{x}(t), \boldsymbol{u}(t))-\boldsymbol{B}^{*}\left(\boldsymbol{x}^{*}(t), \boldsymbol{u}(t)\right)$ which characterizes the non-linearity of DO and observer. In the case of incomplete observability of DO, state vector $x$ and the value of the element $\boldsymbol{\Phi} \boldsymbol{B}(\boldsymbol{x}(t), \boldsymbol{u}(t))$ is unknown. It is making difficult identifying of value of faults. In LDA observer constructed in way to compensate the effect of non-linearity in the residual signal and $\boldsymbol{\Phi} \boldsymbol{B}-\boldsymbol{B}^{*}=0$. However, this condition is fulfilled only when there is no mismatch between the state vectors of DO and observer and $\boldsymbol{e}=0$. To determine the value of fault di which we are interested, feedback by residual signal formed in such way to provide mismatch elimination even in the case of faults should be introduced. 


\section{Introduction of feedback}

After the introduction of feedback by residual signal in the observer his model assumes the form:

$$
\begin{aligned}
& \dot{\boldsymbol{x}}^{*}(t)=\boldsymbol{F}^{*} \boldsymbol{x}^{*}(t)+\boldsymbol{B}^{*}\left(\boldsymbol{x}^{*}(t), \boldsymbol{u}(t)\right)+\boldsymbol{G}^{*} \boldsymbol{u}(t)+\boldsymbol{w}(r)+\boldsymbol{J} \boldsymbol{y}(t) \\
& \boldsymbol{y}^{*}(\mathrm{t})=\boldsymbol{H}^{*} \boldsymbol{x}^{*}(t)
\end{aligned}
$$

where $\boldsymbol{w}(r) \in R^{k}$ is a vector specifying feedback by residual signal.

Taking into account the introduced feedback, change of the mismatch vector will be determined by the equation:

$$
\dot{e}=F^{*} \boldsymbol{e}+\boldsymbol{\Phi B}(\boldsymbol{x}, \boldsymbol{u})-\boldsymbol{B}^{*}\left(\boldsymbol{x}^{*}, \boldsymbol{u}\right)+\boldsymbol{\Phi} \boldsymbol{L} \boldsymbol{d}-\boldsymbol{w}(r)
$$

If formed feedback ensures that the equalities (4) and $\boldsymbol{B}^{*}(\boldsymbol{x}(t), \boldsymbol{u}(t))=\boldsymbol{\Phi} \boldsymbol{B}\left(\boldsymbol{x}^{*}(t), \boldsymbol{u}(t)\right)$ are performed, nonlinearity in the process of identification of faults will be compensated:

$$
\dot{e}=F^{*} \boldsymbol{e}+\Phi \boldsymbol{L} \boldsymbol{d}-\boldsymbol{w}(r)
$$

To ensure the sensitivity of observer to the fault di and the invariance of the rest, matrix $\boldsymbol{\Phi}$ is constructed so that all the columns of matrix $\boldsymbol{\Phi L}$ except ith were zero[1]. Since each column of the matrix L may have only one nonzero element, in the matrix $\mathbf{\Phi} \mathbf{L}$ will be only one element $f_{k}$ not equal to zero. Considering the form of the matrices $\mathrm{F}^{*}$ and properties of matrices $\boldsymbol{\Phi}$ and $\mathbf{L}$ equation (12) can be written as:

$$
\begin{aligned}
& \dot{e}_{1}=e_{2}-w_{1}\left(e_{1}\right), \\
& \dot{e}_{2}=e_{3}-w_{2}\left(e_{1}\right), \\
& \dot{e_{3}}=e_{4}-w_{3}\left(e_{1}\right), \\
& \cdots \\
& \dot{e_{k}}=f_{k} d_{i}-w_{k}\left(e_{1}\right) .
\end{aligned}
$$

The system of equations (13) can be represented as a block diagram (Figure 1) describing the behavior of the residual depending on occurring fault. On the diagram value of the fault arising in the DO presented in the form of the input signal, and estimated value of the fault as an output.

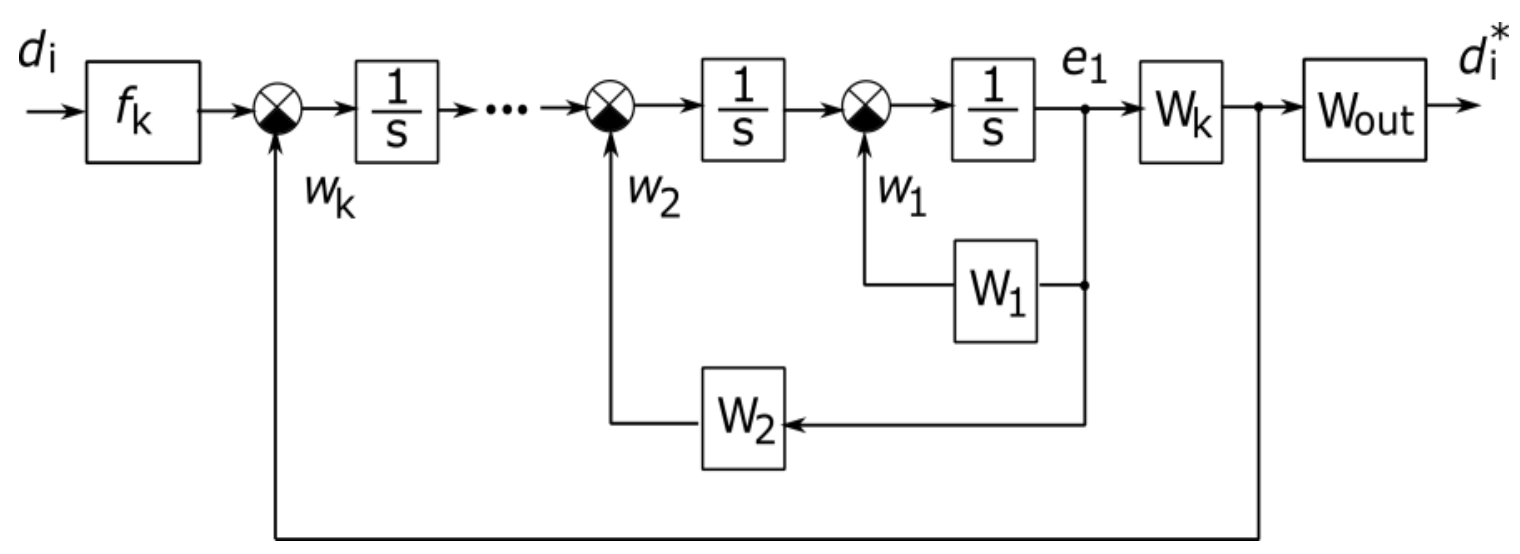

Fig. 1. Block diagram of a diagnostic process relative to the mismatch vector e

To fulfill the condition $\boldsymbol{e} \rightarrow 0$ for a fault of constant size, introducing of integration of the output signal of the open circuit of diagnostic system (Figure 1) can be used:

$$
w_{k}\left(e_{1}\right)=\int\left(w_{k}^{*}\left(e_{1}\right)\right) d t
$$

In this case, the order of equation (13) increases by 1 and becomes $k+1$. 
The next necessary condition to eliminate mismatch between the DO and the observer is to ensure the stability of the observer. The transition process should be damped in time. Is known that for the stability of the system the real parts of all roots of the characteristic equation must be less than 0 .

Writing the system of equations (13) in the form of a differential equation we get:

$$
f_{k} d_{i}=\dot{e}_{k}+w_{k}=\ddot{e}_{k-1}+\dot{w}_{k-1}+w_{k}=\ddot{e}_{k-2}+\ddot{w}_{k-2}+\dot{w}_{k-1}+w_{k}=e_{1}^{(k)}+w_{1}^{(k-1)}+\ldots+w_{k-1}^{(1)}+w_{k}
$$

Accepting elements of the vector of feedback, except the $\mathrm{k}-$ th, proportional to the residual $w_{i}=\frac{T_{i}}{T_{0}} e_{1}(i=1, \overline{1,1})$ it can be obtained:

$$
f_{k} d_{i}==e_{1}^{(k)}+\frac{T_{1}}{T_{0}} e_{1}^{(k-1)}+\ldots+\frac{T_{k-1}}{T_{0}} e_{1}^{(1)}+\int\left(w_{k}^{*}\left(e_{1}\right)\right) d t
$$

By differentiating it can be obtained:

$$
f_{k} d_{i}^{(1)}==e_{1}^{(k+1)}+\frac{T_{1}}{T_{0}} e_{1}^{(k)}+\ldots+\frac{T_{k-1}}{T_{0}} e_{1}^{(2)}+w_{k}^{*}\left(e_{1}\right)
$$

As can be seen, in this equation there is no term with the first derivative of the residual. This is corresponds to the presence of roots of the characteristic equation with real part above zero. To add a missing term in the equation must be:

$$
w_{k}^{*}\left(e_{1}\right)=\frac{T_{k}}{T_{0}} e_{1}^{(1)}+\frac{1}{T_{0}} e_{1}
$$

Then, feedback $w_{\mathrm{k}}(r)$ takes the form:

$$
w_{k}\left(e_{1}\right)=\int\left(\frac{T_{k}}{T_{0}} e_{1}^{(1)}+\frac{1}{T_{0}} e_{1}\right) d t=\frac{T_{k}}{T_{0}} e_{1}+\int \frac{1}{T_{0}} e_{1} d t
$$

The equation (14) takes the form:

$$
T_{0} f_{k} d_{i}^{(1)}=T_{0} e_{1}^{(k+1)}+T_{1} e_{1}^{(k)}+\ldots+T_{k-1} e_{1}^{(2)}+T_{k} e_{1}^{(1)}+e_{1}
$$

Thus, after the introduction of this kind of feedback, residual e1 will tend to zero, after the completion of the transition process, including in the event of faults. This will ensure the synchronization of state vectors of DO and observer.

\section{Determination of values of faults}

The next step of solving the problem is estimation of value of the desired fault. Since the introduction of the feedback was able to arrange that, $\boldsymbol{e}=0$ taking into account (12) it can be written:

$$
\begin{aligned}
& 0=\boldsymbol{\Phi} \boldsymbol{L} \boldsymbol{d}-\boldsymbol{w}(r), \\
& \boldsymbol{w}(r)=\boldsymbol{\Phi} \boldsymbol{L} \boldsymbol{d}
\end{aligned}
$$

Thus, given the form of desired matrixes $\Phi$ and estimation of fault value $d_{i}{ }^{*}$ can be found by the following method:

$$
d_{i}^{*}=\frac{w_{k}\left(e_{1}\right)}{f_{k}}
$$

where $w_{k}\left(e_{1}\right)$ is element of the vector of feedback, $f_{k}$ is element of the ith column of the matrix $\Phi \mathrm{L}$. In view of (15) it can be obtained: 


$$
d_{i}^{*}=\frac{1}{f_{k}}\left(\frac{T_{k}}{T_{0}} e_{1}+\frac{1}{T_{0}} \int e_{1} d t\right)
$$

Given the fact that $e_{1}=0, d_{i}$ fault can be found by integrating the residual $e_{1}$ :

$$
d_{i}^{*}=\frac{1}{f_{k} T_{0}} \int e_{1} d t
$$

Thus the output of the system of identification of faults after the of transient processes will be sought value $d_{i}$. The required system performance can be achieved by selection of $T_{0} \ldots T_{k}$ coefficients.

\section{Example}

Consider nonlinear DO of the third order, described by the following matrixes:

$$
\boldsymbol{F}=\left(\begin{array}{ccc}
0 & 0.01 & 0 \\
0 & -1000 & 200 \\
0 & -5 & -100
\end{array}\right), \quad \boldsymbol{G}=\left(\begin{array}{c}
0 \\
0 \\
250
\end{array}\right), \quad \boldsymbol{H}=\left(\begin{array}{lll}
1 & 0 & 0 \\
0 & 0 & 1
\end{array}\right), \quad \boldsymbol{L}=\left(\begin{array}{ll}
0 & 0 \\
1 & 0 \\
0 & 1
\end{array}\right), \quad \boldsymbol{B}=\left(\begin{array}{c}
0 \\
5 \sin \left(x_{2}\right) \\
0
\end{array}\right)
$$

In this example, the dimension of the output of the system is less than the dimension of the state vector. Thus, only the value of $x_{1}$ and $x_{3}$ are observable and $x 2$ is not observable. Suppose, it is required to build the observer to determine the size of the fault $d_{1}$, acting on 2 nd equation of the system. The observer for the fault $d_{1}$, built with the help of LDA will be described by the following matrix:

$$
\boldsymbol{C}=\left(\begin{array}{ll}
1 & 0
\end{array}\right), \boldsymbol{J}=\left(\begin{array}{cc}
-1000 & 0 \\
0 & 2
\end{array}\right), \boldsymbol{\Phi}=\left(\begin{array}{ccc}
1 & 0 & 0 \\
1000 & 0,01 & 0
\end{array}\right), \boldsymbol{G}^{*}=\left(\begin{array}{l}
0 \\
0
\end{array}\right), \boldsymbol{B}^{*}=\left(\begin{array}{c}
0 \\
0.05 \sin \left(100 x_{2}^{*}-100000 x_{1}^{*}\right.
\end{array}\right)
$$

Model (13) for this case will be:

$$
\begin{aligned}
& \dot{e}_{1}=e_{2}-w_{1}, \\
& \dot{e}_{2}=f_{2} d_{i}-w_{2},
\end{aligned}
$$

where $f_{2}=0.01$.

The equation(17) takes the form:

$$
f_{k} d_{i}^{(1)}=e_{1}^{(3)}+T_{1} e_{1}^{(2)}+T_{2} e_{1}^{(1)}+e_{1}
$$

The conducted research has shown at the synthesis of such observer of the third order is desirable to choose, $T_{0}=\left(\frac{1}{9} T_{c}\right)^{3}, T_{1}=3\left(\frac{1}{9} T_{c}\right)^{2}, T_{2}=3 \frac{1}{9} T_{c}$ where $T_{c}$ is a time at which output signal reaches $5 \%$ closeness of fault value.

If $T_{c}=0.1 \mathrm{~s}$. we obtain:

$$
\begin{aligned}
& T_{0}=1.372 \cdot 10^{-6}, T_{1}=3.7 \cdot 10^{-4}, T_{2}=0.033 \\
& w_{1}=\frac{T_{1}}{T_{0}} e_{1}=270 e_{1}, \quad w_{2}=\frac{T_{1}}{T_{0}} e_{1}+\int \frac{1}{T_{0}} e_{1} d t=24300 e_{1}+729000 \int e_{1} d t
\end{aligned}
$$

To verify the functionality and effectiveness of the proposed method, the modeling of the synthesized observer was carried out. Faults were simulated by introducing a $d_{1}=10$ (Figure 2) and $d_{1}=10 \sin (5 t)$ (Figure 3 ) from $t=5 \mathrm{~s}$, to $t=10 \mathrm{~s}$. and $\mathrm{d}_{2}=5$, from $\mathrm{t}=3 \mathrm{~s}$. 


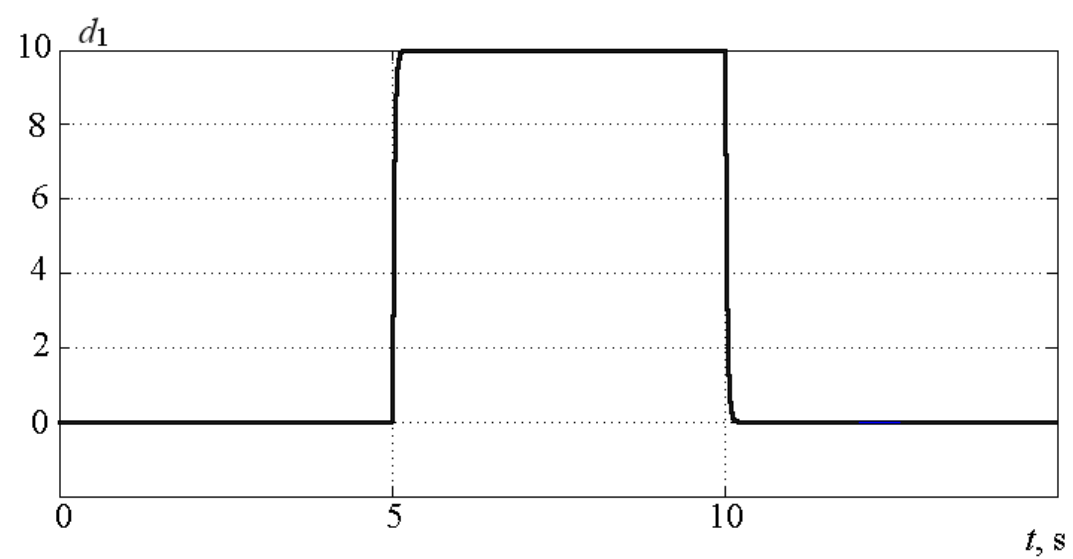

Fig. 2. Output of observer at constant fault value

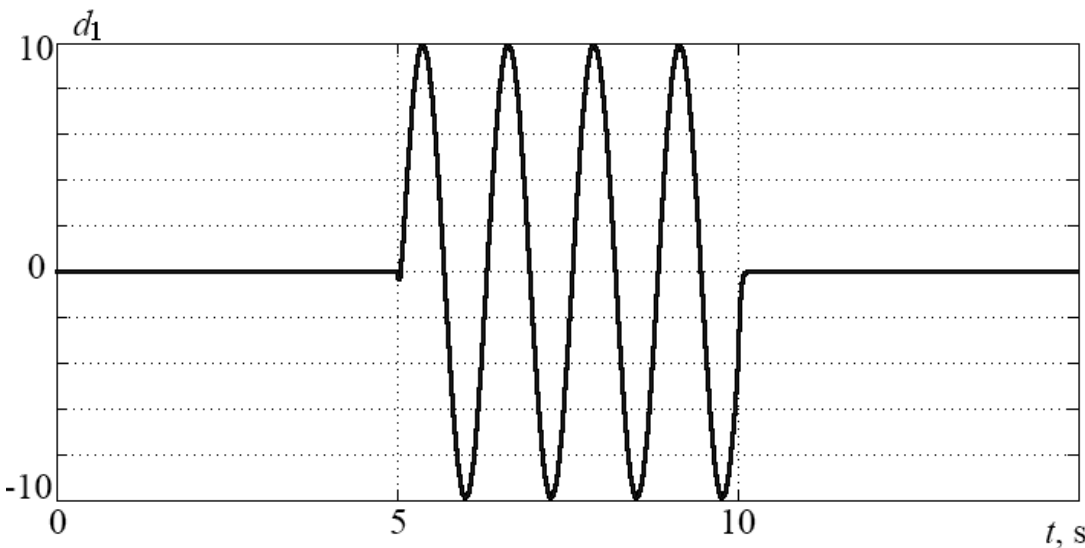

Fig. 3. Output of observer at variable fault value

As can be seen, the system accurately determines the presence or absence of a fault $d_{1}$ and its value. In addition, as required, fault $d_{2}$ does not affect the determination of $d_{1}$. Thus, the results of mathematical modeling is fully confirmed the efficiency and high performance of the proposed method of synthesis of diagnostic observers for identification of faults in nonlinear dynamic systems.

\section{Conclusion}

In this paper, we considered new method of detection, localization and identification of values of faults of the nonlinear dynamic systems. This method consists of applying of logic-dynamic approach for synthesis of diagnostic observers, guaranteeing the independence of the detection and localization of possible faults and introducing of special feedback for diagnostic observers, allowing identifying values of faults. The advantage of this method is the simplicity of implementation and accuracy of the identification of faults. Efficiency of the proposed method of synthesis of diagnostic observers for identification of faults in nonlinear dynamic systems was confirmed by the results of mathematical modeling. The subject of further research will be application of the proposed method for synthesis of the fault accommodation and failsafe control systems for such objects as industrial manipulators and underwater vehicles.

\section{Acknowledgements}

This research was supported by Ministry of education and Science of Russia (project \#14.604.21.0054 from 30.06.14, RFMEFI60414X0054).

\section{References}

[1]. Zhirabok, A. \& Usoltsev, S. Linear methods for fault diagnosis in nonlinear systems, Proc. European Control Conf. ECC'01, Porto, Portugal, 2001 pp. 1363-1366.

[2]. Filaretov V.F., Zirabok A.N., Zuev A.V., Protsenko A. The Development of the Faults Accommodation System for Actuators of Multilink Manipulator // Proc. of the 23rd International DAAAM Symposium, Zadar, Croatia, 2012, pp.575-578. 
[3]. Filaretov V.F., Zhirabok A.N., Zuev A.V., Procenko A.A. Synthesis Method of Fault Tolerant Control System for Manipulators // Journal of Advanced Material Researches. Vol. 717 (2013). P. 551-556

[4]. Isermann R. Fault-Diagnosis Systems: An Introduction from Fault Detection to Fault Tolerance. - Springer-Verlag: Berlin, 2006. 475 p.

[5]. Shumsky A.Ye., Zhirabok A.N. Nonlinear diagnostic filter design: Algebraic and geo-metric points of view - Int. J. Applied Mathematics and Computer Science, 2006, vol. 16, no . 1, pp. 115-127.

[6]. Frank P. Fault diagnosis in dynamic systems using analytical and knowledge-based redundancy - A survey and some new results - Automatica, 1990, vol. 26, pp. 459-474.

[7]. Staroswiecki M., Yang H., Jiang B. Progressive accommodation of aircraft actuator faults - Proc. IFAC Symp. Safeprocess'2006, Beijing. China, 2006, pp. 877-882.

[8]. Siqueira A., M. Terra. A Fault Tolerant Manipulator Robot Based on H2, Hळ and Mixed H2/Hœ Markovian Controls - Proc. of the IEEE International Conference on Control Applications, Taipei, Taiwan, 2004, pp. 309-314.

[9]. Blanke M., Kinnaert M., Lunze J., Staroswiecki M. Diagnosis and Fault Tolerant Control. - Springer-Verlag: Berlin, 2003. 571p.

[10]. Filaretov V., Zhirabok A., Kucher D. New approach to robust observer design, Proc. 19-th Intern. DAAAM Symposium, Slovakia, October, 2008, pp.491-492.

[11]. Dhahri S., Ben Hmida F., Sellami A. LMI-based sliding-mode observer design method for reconstruction of actuator and sensor faults // Int. Journal on Sciences and Techniques of Automatic control. V. 1. №1. 2007. P. 91-107.

[12]. Weng Z., Patton R. Cui P. Active fault-tolerant control of a double inverted pendulum // Proc. IFAC Symp. Safeprocess'2006. Beijing. PR China, 2006. P. 1591-1596.

[13]. Jang B., Staroswiecki M., Cocquempot V. Active fault tolerant control for a class non-linear systems // Proc. IFAC Symp. Safeprocess'03. Washington, USA, 2003. P. 127-132. 